\title{
Pilot surveillance for childhood encephalitis in Australia using the Paediatric Active Enhanced Disease Surveillance (PAEDS) network
}

\author{
P. N. BRITTON ${ }^{1,2,3 *}$, R. C. DALE ${ }^{1,4}$, E. ELLiOTT ${ }^{1,5}$, M. $\operatorname{FESTA}^{1,6}$, \\ K. MACARTNEY $Y^{1,3,7}$, R. BOOY ${ }^{1,2,3,7}$ AND C. A. JONES ${ }^{1,2,3}$ \\ ${ }^{1}$ Discipline of Paediatrics and Child Health, Sydney Medical School, University of Sydney, NSW, Australia \\ ${ }^{2}$ Marie Bashir Institute of Infectious Diseases and Biosecurity Institute (MBI), University of Sydney, NSW, \\ Australia \\ ${ }^{3}$ Department of Infectious Diseases and Microbiology, Children's Hospital at Westmead, NSW, Australia \\ ${ }^{4}$ Department of Neurology, Children's Hospital at Westmead, NSW, Australia \\ ${ }^{5}$ Australian Paediatric Surveillance Unit, Westmead, NSW, Australia \\ ${ }^{6}$ Paediatric Intensive Care Unit, Children's Hospital at Westmead, NSW, Australia \\ ${ }^{7}$ National Centre for Immunisation Research and Surveillance, Westmead, NSW, Australia
}

Received 9 November 2015; Final revision 5 January 2016; Accepted 3 February 2016;

first published online 26 February 2016

\section{SUMMARY}

We aimed to assess the performance of active surveillance for hospitalized childhood encephalitis in New South Wales (NSW) using the Paediatric Active Enhanced Disease Surveillance (PAEDS) network to inform methodology for the nationwide Australian childhood encephalitis (ACE) study. We piloted active surveillance for suspected encephalitis from May to December 2013 at the Children's Hospital at Westmead, Sydney, NSW. Cases were ascertained using four screening methods: weekday nurse screening of admission records (PAEDS), cerebrospinal fluid (CSF) microscopy records, magnetic resonance imaging (MRI) reports, and pharmacy dispensing records. Comprehensive clinical data were prospectively collected on consented participants and subsequently reviewed by an expert panel. Cases were categorized as confirmed encephalitis or 'not encephalitis'; encephalitis cases were sub-categorized as infectious, immune-mediated or unknown. We performed an ICD-10 diagnostic code audit of hospitalizations for the pilot period. We compared case ascertainment in the four screening methods and with the ICD code audit. Forty-eight cases of suspected encephalitis were identified by one or more methods. PAEDS was the most efficient mechanism (yield 34\%), followed by MRI, CSF, and pharmacy audits (yield 14\%,12\%, and $7 \%$ respectively). Twenty-five cases met the criteria for confirmed encephalitis. PAEDS was the most sensitive of the mechanisms for confirmed encephalitis ( $92 \%$ ) with a positive predictive value (PPV) of $72 \%$. The ICD audit was moderately sensitive (64\%) but poorly specific (Sp 9\%, PPV 14\%). Of the 25 confirmed encephalitis cases, 19 (76\%) were sub-categorized as infectious, three (12\%) were immune-mediated, and three (12\%) were 'unknown'. We identified encephalitis cases associated with two infectious disease outbreaks (enterovirus 71, parechovirus 3). PAEDS is an efficient, sensitive and accurate surveillance mechanism for detecting cases of childhood encephalitis including those associated with emerging infectious diseases. Active surveillance significantly increases the ascertainment of encephalitis cases compared with passive approaches.

Key words: Australia, children, encephalitis, surveillance.

\footnotetext{
* Author for correspondence: Dr P. N. Britton, c/o Discipline of Paediatrics and Child Health, The Children's Hospital at Westmead, Locked Bag 4001, Westmead, NSW 2145, Australia.

(Email: philip.britton@health.nsw.gov.au)
} 


\section{INTRODUCTION}

Encephalitis is both a 'marker' syndrome for emerging infectious diseases, and a cause of significant morbidity and mortality in children [1-3]. In contrast to some other countries, encephalitis is not a notifiable condition in Australia with the exception of endemic and imported laboratory-confirmed cases of a few particular viruses known to cause encephalitis; specifically Murray Valley encephalitis virus (MVEV), Australian bat lyssavirus (ABLV) and Japanese encephalitis virus (JEV). Asia has been identified as 'hotspot' for infectious disease emergence [4] and, over the last 20 years, Australasia has seen the identification of several new zoonotic viruses that can cause encephalitis including ABLV and the Henipaviruses [5, 6], with a continuing regional threat from enterovirus 71 (EV71; and other enteroviruses), dengue and chikungunya viruses [5, 7, 8]. Sporadic cases of parasitic meningoencephalitis (e.g. Angiostrongylus cantonensis) may result in death and disability and cause significant public outrage [9]. Furthermore, climate change may increase the range and rate of encephalitis from vector-borne diseases [10] and Australians are travelling at an increasing rate to/from Asian centres [11]. Australia is therefore vulnerable to infectious disease importation and emergence. In addition, encephalitis poses considerable challenges to clinicians because of its protean features, infrequency and severity. We expect early identification and timely description of case clusters/ outbreaks to provide valuable information to both public health authorities, as well as to clinicians, to assist in control efforts and in improving the investigation and management of encephalitis in Australia [12]. Encephalitis it is therefore a priority syndrome for clinical surveillance.

There are few contemporary, prospective studies of childhood encephalitis and none from Australia [1]; those performed, together with retrospective studies, show that children (aged $<10$ years) are at the highest risk of encephalitis, and a higher proportion have an unidentified aetiology (so-called cryptic or unknown encephalitis) compared with adults [1]. Studies from Australia using International Classification of Diseases (ICD)-coded mortality and hospitalization data have also shown that a cause for encephalitis is not identified in the majority of cases, and the highest rates of encephalitis occur in children [13-15]. Some childhood causes of encephalitis are potentially vaccine-preventable (e.g. influenza, varicella, measles) and may represent missed opportunities for primary prevention. Immune-mediated encephalitides (e.g. acute disseminated encephalomyelitis, anti- $N$-methylD-aspartate receptor [16] and anti-voltage gated potassium channel [17]) are increasingly recognized and may be hard to distinguish from infectious encephalitis [18]; in these cases opportunities for early treatment to prevent mortality and neurodevelopmental sequelae may be missed. There is a need for large prospective studies of childhood encephalitis to better define the clinical features and long-term outcome of this challenging condition to inform clinical practice and facilitate discovery of novel infectious and immune-mediated causes in the considerable proportion with unknown aetiology.

The priorities of emerging infectious disease surveillance and outbreak identification as well as the need to address knowledge gaps regarding the epidemiology of childhood encephalitis led us to develop a methodology for active childhood encephalitis surveillance in partnership with the Paediatric Active Enhanced Disease Surveillance (PAEDS) network [19]. Here, we report a pilot study of the methodology performed at the largest paediatric tertiary referral hospital, the Children's hospital at Westmead (CHW) in Sydney, NSW (Australia's most populous state: approximately 1.4 million children aged $0-14$ years). We formally assessed the performance of the surveillance methodology, and this informed the planned rollout of nationwide surveillance-the Australian Childhood Encephalitis (ACE) study. Furthermore, we discuss the potential of PAEDS-ACE surveillance as a platform for clinical research.

\section{METHODS}

The CHW is a 250-bed tertiary/quaternary paediatric hospital in Sydney, NSW, Australia. It is one of the largest children's hospitals in Australia, and the site of the national coordinating centre for the PAEDS network (www.paeds.edu.au) [19]. We undertook pilot prospective surveillance for suspected encephalitis at CHW from 1 May to 1 December 2013. The study was approved by the Sydney Children's Hospitals Network (SCHN) human research ethics committee (approval no. HREC/13/SCHN/402).

PAEDS nurses systematically identified suspected encephalitis cases by screening of hospital admissions (emergency department, inpatient wards, paediatric and neonatal intensive care) for key words (see Table 1). Additionally, potential cases were identified 
Table 1. Screening definitions for suspected encephalitis implemented at the Children's Hospital at Westmead, 1 May to 1 December 2013

Screening methodologies

1. Admission screen using key words by PAEDS research nurses:

Daily screen of hospital admission line listings (emergency department, wards, intensive care)

Encephalopathy/encephalitis Behavioural change

Personality change Altered (level of) conscious state

Irritability Abnormal head imaging

Seizures $\quad$ Focal neurological signs

ADEM Lethargy

2. Cerebrospinal fluid screening (CSF)

- Weekly review of all CSF specimens submitted to the hospital diagnostic laboratory

- Screening chart review of the admission associated with a CSF microscopy result showing $\geqslant 1$ (x10 $6 / 1)$ poly-morpho-nuclear $(\mathrm{PMN})$ cell $\mathrm{OR} \geqslant 5\left(\mathrm{x} 10^{6} / 1\right)$ white cell count $(\mathrm{WCC})$

- We excluded specimens labelled as being performed for routine oncology testing

3. Neuroimaging screening

- Weekly review all brain or spine magnetic resonance imaging (MRI) reports of those scans performed at the Children's Hospital at Westmead

- Screening chart review of those with imaging requests AND/OR reports containing the key words encephalopathy OR encephalitis

4. Pharmacy dispensing records screening

- Monthly review of hospital pharmacy dispensing records for acyclovir dispensed to inpatients

- Screening chart review of all hospitalisations in which a patient was dispensed acyclovir by the pharmacy

'Suspected encephalitis' case definition* applied for case inclusion during screening chart review

Inclusion criteria

1. Age $0-14$ years AND;

2. Hospitalized with acute encephalopathy (defined by altered level of consciousness, lethargy and/or personality change lasting $\geqslant 24 \mathrm{~h}) \mathrm{AND}$;

3. Has one or more of the following: fever, seizures, focal neurological findings, at least one abnormality of cerebrospinal fluid (CSF; age-determined pleocytosis, or elevated protein $\geqslant 40 \mathrm{mg} / \mathrm{dl}$ ), or EEG/neuroimaging findings consistent with infection-related encephalitis.

Exclusion criteria

- Viral aseptic meningitis without encephalopathy

- Acute bacterial meningitis without cerebral involvement

- Non-infectious CNS disorders due to hypoxic/ischaemic, vascular, toxic and metabolic causes

- CNS disorders lasting $<24 \mathrm{~h}$

- Chronic encephalopathies

ADEM, Acute disseminated encephalomyelitis.

* Based upon the Brighton criteria and the California encephalitis project case definitions [20, 31].

by screening of: cerebrospinal fluid (CSF) microscopy records for an elevated neutrophil or total white cell count; magnetic resonance imaging (MRI) brain/spine reports for encephalopathy/encephalitis; and pharmacy records for inpatients who were dispensed acyclovir (see Table 1). Where a potential case was identified using one or more of these screening approaches the available patient records (emergency department summary, clinical notes, laboratory and medical imaging records) were reviewed as to whether the case met the suspected encephalitis surveillance definition (i.e. met inclusion criteria, and did not meet any exclusion criteria: see Table 1). Consent was sought from the parent/guardian of the child to be interviewed as well as review of the clinical records in order to complete a case report form. We collected data including: demographics, risk factors (e.g. travel, animal exposures), clinical features, laboratory testing, neuroimaging, medications, hospitalization details (level of care, length of stay), outcome at discharge, and admission ICD-10 coding. This study was observational and the investigation and management of cases was determined by treating physicians without direct input from the PAEDS/ACE investigator team.

The comprehensive data from these cases were subsequently reviewed approximately 3 months postadmission date by an expert panel comprising a 


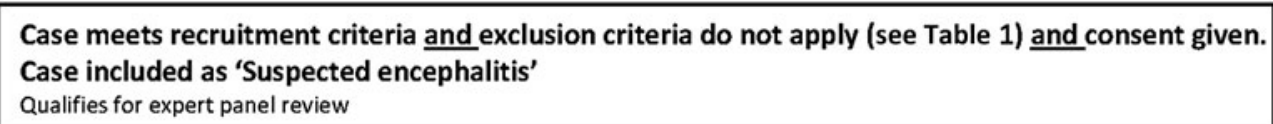
$\downarrow$

\section{Expert panel data}

- Full access to data collected using PAEDS-ACE case report form: includes demographic, risk factor, clinical features, laboratory testing, neuroimaging, medications, hospitalisation details (level of care, length of stay), outcome at discharge, admission ICD-10 coding.

- Additional access to hospital discharge summary, neuroimaging reports, electro-encephalogram (EEG) reports.

- Where necessary, original neuroimaging reviewed, attending clinicians contacted.

\begin{tabular}{|c|c|c|c|}
\hline \\
\hline \multicolumn{4}{|c|}{ Brighton and IEC encephalitis case definitions (CD) applied and case categorised } \\
\hline \multicolumn{2}{|l|}{$\downarrow$} & \multicolumn{2}{|c|}{$\downarrow$} \\
\hline \multicolumn{2}{|c|}{$\begin{array}{l}\text { Case meets encephalitis CD: } \\
\text { - Includes those cases classified as Brighton Level } 2 \\
\text { encephalitis AND/OR IEC confirmed or probable } \\
\text { encephalitis. } \\
\text { - Includes those cases classified as Brighton Level } 3 \\
\text { encephalitis AND IEC possible encephalitis. }\end{array}$} & \multirow{3}{*}{\multicolumn{2}{|c|}{$\begin{array}{l}\text { Case did not meet encephalitis CD: } \\
\text { - Either case definition is not met. } \\
\text { OR } \\
\text { Case determined to have a confirmed } \\
\text { alternative diagnosis } \\
\text { OR } \\
\text { Specific 'encephalitis mimics' excluded } \\
\text { - Acute cerebellar syndromes. } \\
\text { Bacterial meningitis including tuberculous } \\
\text { meningitis without neuroimaging evidence of } \\
\text { OR brain parenchymal inflammation. } \\
\text { Inadequate testing available to apply CD }\end{array}$}} \\
\hline$\downarrow$ & & & \\
\hline \multirow{3}{*}{\multicolumn{2}{|c|}{$\begin{array}{l}\text { Case categorised as 'Encephalitis' } \\
\text { - Full review of microbiological, autoimmune testing } \\
\text { available. } \\
\text { - Where an infectious agent is identified as } \\
\text { associated with the acute illness directly (culture, } \\
\text { NAAT) or indirectly (serology), the case is } \\
\text { categorised as 'infectious encephalitis'. } \\
\text { - Where a defined immune-mediated encephalitis } \\
\text { (ADEM, Ab-mediated (e.g. anti-NMDAR)) is } \\
\text { identified, the case is categorised as 'immune- } \\
\text { mediated encephalitis'. } \\
\text { Where a case is categorised as 'encephalitis' case } \\
\text { but does not fall under either points } 2 \text { or 3, the case } \\
\text { is categorised as 'unknown encephalitis'. }\end{array}$}} & & \\
\hline & & & $\downarrow$ \\
\hline & & \multicolumn{2}{|c|}{$\begin{array}{l}\text { Case categorised as 'Not encephalitis' } \\
\text { Confirmed alternative diagnoses recorded (including } \\
\text { discharge summary clinical diagnoses where no } \\
\text { confirmed pathological/microbiological diagnosis } \\
\text { defined). } \\
\text { Those cases with inadequate testing available to apply } \\
C D \text { annotated as such. }\end{array}$} \\
\hline$\downarrow$ & & & $\downarrow$ \\
\hline $\begin{array}{l}\text { 'Infectious encephalitis' } \\
\text { Identified pathogens categorised as to } \\
\text { their likely causative role as confirmed, } \\
\text { probable or possible according to the } \\
\text { Granerod et al criteria. }\end{array}$ & \multicolumn{2}{|c|}{$\begin{array}{l}\text { 'Immune-mediated encephalitis' } \\
\text { ADEM cases sub-categorised using the } \\
\text { Brighton criteria. } \\
\text { Where a non-ADEM, non-antibody } \\
\text { mediated but immune pathology is } \\
\text { postulated it is categorised as an other } \\
\text { immune-mediated encephalitis }\end{array}$} & 'Unknown encephalitis'. \\
\hline
\end{tabular}

Fig. 1. Flowchart specifying Australian Childhood Encephalitis (ACE) study expert panel process of case categorization. ADEM, Acute disseminated encephalo-myelitis; PAEDS, Paediatric Active Enhanced Disease Surveillance network.

paediatric infectious diseases (ID) physician, paediatric neurologist, paediatric clinical microbiologist, and paediatric ID epidemiologist. The panel categorized the cases as confirmed encephalitis or 'not encephalitis' using the Brighton and international encephalitis consortium (IEC) encephalitis case definitions [20, 21] (see Fig. 1). Confirmed encephalitis cases were sub-categorized as infectious, immune-mediated, and 'unknown'. Of the infectious cases, where a pathogen was identified on acute diagnostic testing, the panel categorized it as a confirmed, probable or possible cause of the presentation according to the criteria of Granerod et al. [22] (see Fig. 1). There was a subgroup of cases that met the 'suspected encephalitis' case definition but could not be definitively further categorized as they did not have adequate investigations, i.e. no central nervous system (CNS) imaging, nor an electro-encephalogram (EEG) performed. The panel was therefore unable to fully assess the features of CNS inflammation and apply the clinical case 
Table 2. International Classification of Diseases version 10 (ICD-10) diagnostic codes used to extract admissions with encephalitis and encephalitis associated conditions

\begin{tabular}{|c|c|c|c|}
\hline Condition & $\begin{array}{l}\text { ICD-10 } \\
\text { code }\end{array}$ & Condition & ICD-10 code \\
\hline $\begin{array}{l}\text { Tuberculous } \\
\text { meningo-encephalitis }\end{array}$ & A17.8 & HHV6, other herpes viral encephalitis & $\mathrm{B} 10.0$ \\
\hline Neurosyphilis & $\begin{array}{l}\text { A } 50.4 \\
\text { A } 52.1\end{array}$ & Measles and rubella encephalitis & B05.0, B06.0 \\
\hline SSPE, CJD & $\begin{array}{r}\text { A81.1, } \\
\text { A81.9 }\end{array}$ & HIV & B20.x \\
\hline Rabies & A82.x & Mumps encephalitis & $\mathrm{B} 26.2$ \\
\hline $\begin{array}{l}\text { Mosquito-borne viral } \\
\text { encephalitis }\end{array}$ & A83.x & Cerebral cryptococcosis & B45.1 \\
\hline Tick-borne encephalitis & A84.x & Chagas, African trypanosomiasis & B57.4, B56.x \\
\hline Other viral encephalitis & A $85 . x$ & Toxoplasma meningo-encephalitis & B58.2 \\
\hline Unspecified viral encephalitis & A86 & Amoebic meningo-encephalitis & B 60.2 \\
\hline $\begin{array}{l}\text { Other specified viral infection } \\
\text { of CNS }\end{array}$ & A 88.8 & ADEM & G04.0 \\
\hline $\begin{array}{l}\text { Unspecified viral infection } \\
\text { CNS }\end{array}$ & A89 & $\begin{array}{l}\text { Bacterial meningo-encephalitis NOS, ANE, Other specified } \\
\text { encephalitis, Encephalitis NOS }\end{array}$ & $\begin{array}{l}\mathrm{G} 04.2, \mathrm{G} 04.8, \\
\mathrm{G} 04.9\end{array}$ \\
\hline West Nile virus & A92.3 & Encephalitis/EM in diseases classified elsewhere & G05.x \\
\hline $\begin{array}{l}\text { Herpes simplex virus } \\
\text { encephalitis }\end{array}$ & B00.4 & Unspecified encephalopathy (including influenza) & G93.4 \\
\hline Varicella encephalitis & B01.1 & Sequelae of viral encephalitis & B94.1 \\
\hline Zoster encephalitis & $\mathrm{B} 02.0$ & Sequelae of infectious and parasitic disease & B94.8 \\
\hline
\end{tabular}

ADEM, Acute disseminated encephalo-myelitis; ANE, acute necrotizing encephalitis; CJD, Creutzfeld-Jacob disease; CNS, central nervous system; EM, encephalomyelitis; HHV, human herpesvirus 6; HIV, human immunodeficiency virus; NOS, not otherwise specified; SSPE, sub-acute sclerosing pan-encephalitis.

definitions. We have chosen to categorize these cases as 'not encephalitis', but to indicate that incomplete testing occurred and that they could not be definitively categorized.

In applying the IEC/Brighton definitions of encephalitic young infants [20,21], the following adjustments were made: lethargy was allowed as part of the inclusion criteria when it was reported as a sign by a clinician; irritability in young children was not considered to be a feature of encephalopathy.

We compared the PAEDS approach to case ascertainment of suspected and confirmed encephalitis with screening by CSF, MRI, and pharmacy record methods. We defined the yield of screening to be the number of cases of suspected encephalitis identified as a proportion of the number of patient records screened. Using the expert panel diagnosis of encephalitis as a 'gold-standard' for confirmed encephalitis and the total number of confirmed cases (detected using any of the four methods), we calculated the sensitivity, specificity and positive predictive (PPV) value of each of the four different surveillance approaches. In addition, we performed an ICD-10 code audit of all hospitalizations for the pilot period using encephalitis-associated ICD-10 diagnostic codes (see Table 2). We compared PAEDS identification (active surveillance) of suspected and confirmed encephalitis with the ICD-code audit (passive surveillance).

\section{RESULTS}

We identified 48 cases of suspected encephalitis by one or more mechanisms over the 7-month pilot period; more than six cases per month or $2 \cdot 5 / 1000$ admissions (average 2700 admissions/month). The total numbers of cases screened and identified by the different surveillance mechanisms are shown in Table 3. The use of dedicated surveillance nurses (PAEDS screening) was the most efficient mechanism for case ascertainment with 34 cases identified from 89 charts reviewed (38\% yield), followed by MRI audit (19 from 138 reviewed, $14 \%$ yield), CSF audit (30 from 259 reviewed, 12\% yield), and pharmacy records (11 from 158 reviewed, 7\% yield). In addition to the 34 cases identified by PAEDS, the CSF audit identified 12 additional unique cases of suspected encephalitis 
Table 3. Screening yield, sensitivity, specificity and positive predictive value of piloted screening methodologies for childhood encephalitis at the Children's Hospital at Westmead, 1 May 1 December 2013

\begin{tabular}{llllll}
\hline \hline & PAEDS & MRI & CSF & Pharmacy & Total unique \\
\hline Cases screened & 89 & 138 & 259 & 158 & n.a. \\
Cases suspected encephalitis (SE) identified & 34 & 19 & 30 & 11 (1) & 48 \\
& $(7$ unique) & $(1$ unique) & $(12$ unique) & $(1$ unique) & \\
Screening yield (SE/screened) & $38 \%$ & $14 \%$ & $12 \%$ & $7 \%$ & n.a. \\
Consent obtained (\% SE) & $32(94)$ & $18(95)$ & $24(80)$ & $8(73)$ & $40(83)$ \\
Cases of confirmed encephalitis & 23 & 15 & 15 & 8 & 25 \\
Sensitivity & $92 \%$ & $60 \%$ & $60 \%$ & $32 \%$ & $100 \%$ \\
Specificity & $40 \%$ & $80 \%$ & $40 \%$ & $100 \%$ & n.a. \\
PPV & $72 \%$ & $83 \%$ & $63 \%$ & $100 \%$ & $63 \%$ \\
\hline \hline
\end{tabular}

CSF, Cerebrospinal fluid; MRI, magnetic resonance imaging; n.a., not applicable; PAEDS, Paediatric Active Enhanced Disease Surveillance network; PPV, positive predictive value; SE, suspected encephalitis.

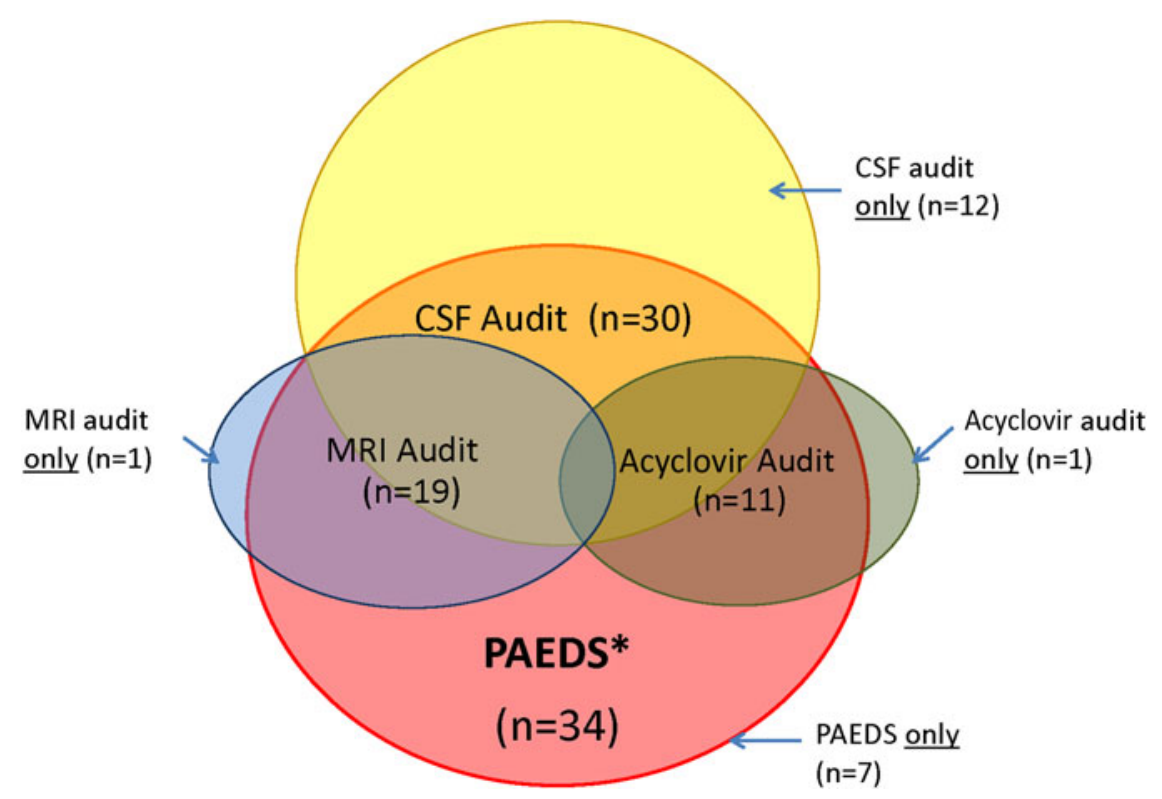

Fig. 2. Venn diagram showing suspected encephalitis case ascertainment using four ascertainment mechanisms. Diagram intersection numbers: PAEDS + CSF + MRI $(n=8)$; PAEDS + CSF + acyclovir $(n=5)$; PAEDS + MRI + acyclovir $(n=0)$; PAEDS + CSF $(n=2) ;$ PAEDS + MRI $(n=7) ;$ PAEDS + acyclovir $(n=2)$. CSF, Cerebrospinal fluid; MRI, magnetic resonance imaging; PAEDS, Paediatric Active Enhanced Disease Surveillance network.

(Fig. 2); while MRI and pharmacy screens identified one unique case each (Fig. 2). Of the additional 12 unique cases identified by CSF audit, 10 were young infants with suspected viral meningo-encephalitis.

A total of $40 / 48$ cases of suspected encephalitis ascertained by the four screening methods consented to participate in the cohort study. Following expert panel review, $15(38 \%)$ of the 40 cases were classified 'not encephalitis'. Five of these cases were young infants (aged $<12$ months) with enterovirus or parechovirus infection (four CSF PCR positive) who did not have MRI or EEG performed and were thus considered as 'not encephalitis' albeit on the basis of an incomplete investigation. Four of these five cases were identified by CSF screening. Notably, the majority of 'not encephalitis' cases were diagnosed with other infectious and/or inflammatory CNS syndromes (e.g. aseptic meningitis, myelitis, clinically isolated syndrome - results not shown). We therefore identified 25 confirmed encephalitis cases over the 7-month pilot period; more than three cases per month or $1 \cdot 3 / 1000$ admissions. Of these, $19(76 \%)$ were sub-categorized as infectious, three $(12 \%)$ were immune-mediated, and three $(12 \%)$ were 'unknown' (Table 4$)$. Of the 
Table 4. Suspected encephalitis case categorisation, age and sex following review by Australian Childhood Encephalitis study expert panel at the Children's Hospital at Westmead, 1 May to 1 December 2013

\begin{tabular}{|c|c|c|c|c|c|}
\hline & \multirow{2}{*}{$\begin{array}{l}\text { Suspected } \\
\text { encephalitis } \\
(n=40)\end{array}$} & \multicolumn{3}{|c|}{ Confirmed encephalitis $(n=25)$} & \multirow[b]{2}{*}{$\begin{array}{l}\text { 'Not-encephalitis'* } \\
(n=15)\end{array}$} \\
\hline & & $\begin{array}{l}\text { Infectious } \\
(n=19)\end{array}$ & $\begin{array}{l}\text { Immune-mediated } \\
(n=3)\end{array}$ & $\begin{array}{l}\text { Unknown } \\
(n=3)\end{array}$ & \\
\hline $\begin{array}{l}\text { Age, years, median } \\
\text { (range) }\end{array}$ & $\begin{array}{l}2 \cdot 3 \\
(0 \cdot 0-13 \cdot 3)\end{array}$ & $\begin{array}{l}2 \cdot 0 \\
(0 \cdot 0-9 \cdot 3)\end{array}$ & $\begin{array}{l}5 \cdot 6 \\
(3 \cdot 5-7 \cdot 6)\end{array}$ & $\begin{array}{l}4 \cdot 9 \\
(2 \cdot 0-13 \cdot 3)\end{array}$ & $\begin{array}{l}0 \cdot 8 \\
(0 \cdot 0-13 \cdot 3)\end{array}$ \\
\hline Male sex & $50 \%$ & $50 \%$ & $67 \%$ & $67 \%$ & $43 \%$ \\
\hline
\end{tabular}

* Five of these 15 cases were young infants (aged $<12$ months) with enterovirus or parechovirus infection who did not have adequate CNS imaging or EEG performed. The panel was therefore unable to fully assess the features of CNS inflammation and apply the clinical case definitions. In these cases incomplete testing occurred and they could not be definitively categorized, i.e. 'not encephalitis' - incomplete testing.

Table 5. Results of pilot International Classification of Diseases (ICD) diagnostic code hospital admission audit for encephalitis and encephalitis associated conditions at the Children's Hospital at Westmead, 1 May to 1 December 2013

\begin{tabular}{llll}
\hline \hline & $\begin{array}{l}\text { Full ICD-10 } \\
\text { code audit }\end{array}$ & $\begin{array}{l}\text { ICD-10 code audit } \\
\text { excluding G93.4* }\end{array}$ & $\begin{array}{l}\text { ICD-10 code audit excluding } \\
\text { G93.4, B94.1 and B94.8* }\end{array}$ \\
\hline Cases identified & 115 & 51 & 40 \\
Cases suspected encephalitis (SE) identified & 25 & 20 & 20 \\
Consent obtained (\% SE) & $23(92 \%)$ & $20(100 \%)$ & $20(100 \%)$ \\
Cases of confirmed encephalitis & 16 & 13 & 13 \\
Sensitivity & $64 \%$ & $52 \%$ & $52 \%$ \\
Specificity & $9 \%$ & $21 \%$ & $27 \%$ \\
PPV & $14 \%$ & $25 \%$ & $33 \%$ \\
\hline \hline
\end{tabular}

PPV, Positive predictive value; SE, suspected encephalitis.

* G93.4, Unspecified encephalopathy (including influenza); B94.1, sequelae of viral encephalitis; B94.8, sequelae of infectious and parasitic disease.

cases categorized as infectious encephalitis, $79 \%$ were associated with one of three pathogens: enteroviruses (including Ev71), influenza or human parechovirus.

The sensitivity, specificity and PPV value of the different surveillance mechanisms are shown in Table 3. The PAEDS mechanism had the highest sensitivity for confirmed encephalitis $(92 \%)$ with a specificity of $40 \%$ and PPV of $72 \%$. The pharmacy dispensing records screen had the highest specificity $(100 \%)$, but was insensitive $(32 \%)$. Combining PAEDS ascertainment with CSF screening would result in all confirmed encephalitis cases being identified (sensitivity 100\%), but would result in reduced specificity (all 15 'not encephalitis' cases also identified). Only a small number of unique cases were identified by MRI and pharmacy ascertainment (one each) and neither were able to be contacted to consent to expert panel review. Unfortunately, analysing these methodologies in combination with PAEDS was therefore not possible.
Current information about encephalitis hospitalizations in Australia relies upon analyses of national hospitalization datasets [13-15]. We therefore undertook an audit of International Classification of Diseases-Australian Modification-10 (ICD-AM-10) discharge diagnostic codes for the pilot surveillance period in NSW. This audit showed that if all encephalitis-associated codes were included, the sensitivity of this passive use of diagnostic-coded medical record data for encephalitis surveillance is $64 \%$ compared to active surveillance and it is poorly specific $(9 \%$, Table 5). The total number of suspected encephalitis cases ascertained by active (PAEDS) surveillance exceeded that ascertained by passive (ICD audit) surveillance (32 vs. 25 cases). There were no suspected encephalitis cases ascertained by the ICD audit that were not ascertained by PAEDS. The specificity of the coding audit could be improved by removing the least specific ICD-10 encephalitis-associated 
codes (G93.4, B94.1, B94.8); however, this resulted in a lower sensitivity.

\section{DISCUSSION}

We have reported this evaluation of pilot surveillance for childhood encephalitis in Australia to show that the use of active hospital-based surveillance using specialized nurses - the PAEDS platform - is an efficient mechanism for the detection of childhood suspected encephalitis. It is also highly sensitive for the detection of confirmed encephalitis cases that have been diagnosed rigorously by an expert panel of clinicians using contemporary consensus case definitions for encephalitis $[20,21]$. Our active surveillance increased case ascertainment of suspected and confirmed encephalitis cases compared to passive approaches such as retrospective review of ICD hospital discharge codes, which are relatively insensitive and nonspecific. Furthermore we have identified very young infants to be a particularly challenging group to identify and assess, an important insight given the likely highest incidence of encephalitis in the very young. Moreover, prospective case identification allows for additional data, often poorly recorded in the medical record (if available), to be gathered from parents/ carers.

Our pilot childhood encephalitis surveillance study methodology compares favourably with methods for adult encephalitis surveillance that have recently been published by Eastwood et al. from a regional adult referral hospital in northern NSW [23]. In this study, 'suspected encephalitis' cases were identified using three ascertainment approaches and their performance was similarly assessed using an expert review panel as a 'gold standard' for diagnosis. Of those patients meeting the surveillance (screening) definitions, $18 \%$ (20/113) met the more specific review panel definition and 35\% (7/20) were diagnosed as confirmed encephalitis (13/20 had confirmed alternative diagnoses) [23]. Analogous figures from our work for PAEDS include a screening yield of $34 \%$ and PPV of $72 \%$ (Table 3). By comparison MRI and pharmacy screening (acyclovir dispensed) were associated with higher PPV, but much lower screening yield and poor sensitivity. Given our stated priority that encephalitis surveillance be used to detect emerging infectious disease outbreaks, the methodology employed requires high sensitivity. PAEDS appears to perform with this capacity. It is not highly specific and would likely result in false-positive detections of encephalitis activity, but when coupled with timely detailed review of cases as presented here, we suggest that these false positives can be readily identified. This approach, however, is resource intensive and best suited to sentinel site surveillance rather that universal surveillance. We have noted that surveillance sensitivity is maximized when PAEDS is combined with additional CSF screening. We would also suggest that CSF screening, based upon the additional cases that it identified in this pilot, could be restricted to young children ( $<12$ months only).

Active surveillance for childhood encephalitis using the ACE study methodology does indeed appear to have considerable potential as a tool for identifying emerging infectious diseases. The period of pilot surveillance overlapped with two large epidemics of neuro-tropic viruses in metropolitan Sydney. The first, between February and June 2013, caused by EV71 resulted in a large number presentations to hospital with hand, foot and mouth disease and admissions with neurological complications including aseptic meningitis, polio-like acute flaccid paralysis, encephalomyelitis and encephalitis [7]. The second, between October 2013 and February 2014, caused by human parechovirus genotype 3 (HPeV3) resulted in a large number of admissions with sepsis-like disease and meningo-encephalitis in young children $[24,25]$. The ACE study captured cases associated with both these epidemics and contributed to characterizing the severe end of the disease spectrum. The real-time identification of encephalitis cases using the PAEDS platform offers a significant advantage compared with alternatives by maximizing the potential of surveillance to improve outbreak responsiveness including individual case management and public health action.

The poor sensitivity and specificity of coded hospital discharge diagnoses for confirmed encephalitis identified in this study highlights the limitation of using this data to answer key questions regarding the epidemiology of childhood encephalitis. This finding contrasts with the analysis of Bernard et al. from France [26]. It is possible that the limitations of coded data are particularly evident for childhood encephalitis where the spectrum of aetiologies is different to those in adults.

Our study has also highlighted that very young children/infants pose a challenge both for clinicians and encephalitis researchers in terms of defining the specific features of encephalopathy. The key words 'lethargy' and 'irritability' were used in screening for 
CNS dysfunction. In a recently published retrospective study, we note neither feature was used to define encephalopathy [27]. We found that these key words frequently identified a subset of mainly young infants who were found to have confirmed alternative diagnoses (viral respiratory tract infections, urinary tract infections) and were therefore excluded. However, a minority of children were not excluded and the application of current IEC/Brighton encephalitis definitions to this subgroup was difficult. Lethargy, reported as a symptom by parents/carers, did not appear to have a well-defined threshold and was nonspecific. The expert panel therefore resolved to use only clinician-identified lethargy and required it be present for $>24 \mathrm{~h}$ as specified in the IEC/Brighton definitions; we acknowledge that the reliability of reporting this sign is also unclear. Furthermore, the reliability of irritability as an indicator of personality/behavioural change in a baby, in the absence of an alternative cause has not been validated. The expert panel resolved that irritability in young infants was too non-specific to be formally considered a feature of encephalopathy. One approach to addressing these challenges would be to ensure detailed follow-up of both the encephalitis and 'not encephalitis' cases to determine the proportion of children with sequelae, as we propose to do in the nationwide ACE study. Defining the clinical 'risk factors' associated with adverse outcomes may assist in refining clinical case definitions of encephalitis in young infants.

The challenge posed by infants is also highlighted by the additional yield of CSF screening to PAEDS key word screening in this age group. CSF screening identified a notable proportion of additional unique cases of suspected encephalitis. Most of these cases were suspected viral meningo-encephaltis caused by enterovirus or parechovirus (results not shown). It is likely that optimal case ascertainment of suspected encephalitis in young infants requires the addition of CSF screening to PAEDS, but it is possible that this additional yield may be confined to the epidemic activity of both EV71 and HPeV3 which overlapped with the surveillance pilot.

Interestingly, we would note a trend towards a difference in age between infectious and immunemediated encephalitis in this pilot. Although this is based on only a very small number of immunemediated cases, and the age ranges of the groups overlap, we consider this a potentially important trend. It needs to be evaluated in the larger cohort that is being established.
The proportion of suspected encephalitis cases determined by the expert panel to be 'not encephalitis' is higher than that in a similarly designed, prospective study across all ages from the UK [28]; however, in contrast to that study, the majority of 'not encephalitis' cases in this pilot were CNS infections, or infection-associated CNS inflammatory disorders. The potential overlap in aetiology and pathogenesis of conditions that mimic encephalitis (i.e. cases in the 'not encephalitis' group) with cases of confirmed encephalitis in children requires further investigation. Although only a small proportion of encephalitis cases had no possible aetiology assigned, the diagnostic testing in these cases was often incomplete (results not shown). This highlights the need for wider dissemination of standardized diagnostic algorithms for encephalitis in adults and children, such as those recently published by our group for Australia and by the IEC [21, 29, 30].

Our pilot study was limited by the human research ethics committee requirement for consent from ascertained suspected encephalitis cases for collection and review of clinical data by the expert panel. As a result, our current estimates from both surveillance and ICD-code audit performance against confirmed encephalitis are limited by the exclusion of some cases of suspected encephalitis. This has now been addressed by the PAEDS network which enables collection of a minimum level of data from the medical record using a 'waiver of consent' since January 2014; additional data can still be sought using parent/carer interview with consent. We acknowledge that we have not accounted for the relative costs incurred by each surveillance method; however, we note that by including childhood encephalitis surveillance within the framework of PAEDS, an economies of scale is achieved because PAEDS nurses also conduct screening and case ascertainment for up to 4-6 other conditions simultaneously, thus maximizing efficiency of time and outputs [19]. Evaluation of the pilot is also limited by its performance at a single site. The PAEDS network comprises five geographically distinct, tertiary paediatric hospitals across Australia in which screening by nurses is performed in the context of different admission policies and clinical information technology systems. The performance of the ACE study methodology requires continual evaluation as it implemented nationwide across the network.

Our evaluation has informed the rollout of national childhood encephalitis surveillance that began in 2014 
and potentially across all age groups in Australia in the future. Up to August 2015, following rollout of PAEDS-ACE surveillance to all five sites, data on 225 suspected encephalitis cases has been gathered for analysis. The screening yield nationally has been consistent with that presented in this analysis (P. N. Britton, unpublished results). PAEDS-ACE surveillance is increasingly realizing its potential to contribute to outbreak responsiveness by regularly reporting case numbers to jurisdictional public health authorities, including any increased activity of individual infectious aetiologies, and providing clinical advice where requested. Furthermore, this surveillance platform is central to establishing a cohort of childhood encephalitis cases for clinical epidemiological analysis and neuro-developmental follow-up to address several key unanswered questions. We have developed and now disseminated through the PAEDS network a standardized diagnostic algorithm to maximize the identification of a confirmed or probable aetiological diagnosis using currently available tests [29]. The ACE study also salvages laboratory specimens from identified cases. These specimens-in particular those from children with 'unknown' encephalitis will be stored and later subjected to high throughput, molecular technologies to identify undetected known and potentially novel causes of infectious encephalitis. Remaining specimens from children with encephalitis will be archived for future research into the aetiology and pathogenesis of this disease.

\section{CONCLUSIONS}

PAEDS is an efficient, sensitive and accurate surveillance mechanism for detecting cases of childhood encephalitis including those associated with emerging infectious diseases. It performs favourably compared to other methods of encephalitis case ascertainment. In addition, it has the advantage of real-time identification of suspected cases. This, coupled with detailed, expert clinical review is resource intensive but highly reactive and has the potential to detect outbreaks and inform clinical and public health responses. Active surveillance significantly increases the ascertainment of encephalitis cases compared with passive approaches and, in time, will establish an important cohort of childhood encephalitis to better understand the clinical epidemiology and outcome of this challenging condition.

\section{ACKNOWLEDGEMENTS}

We thank the PAEDS nurses who substantially contributed to the implementation of the surveillance protocol, Jocelynne McRae and Jenny Murphy. We also thank the other PAEDS investigators from the Children's Hospital at Westmead, Dr Nicholas Wood and Professor Peter McIntyre.

The ACE study is funded by grants from the Australian Department of Health (Surveillance branch, DoHA3931213), and the National Health and Medical Research Council (NHMRC) of Australia Centre for Research Excellence (CRE) in Critical Infections (1 001021 ); both to C.A.J. and R.B.

P.N.B. is supported by a NHMRC post-graduate scholarship (1 074 547), the Royal Australasian College of Physicians (RACP) NHMRC Award for Excellence, and Norah Therese-Hayes/Sydney Medical School Dean's paediatric infectious diseases fellowship. E.E. is supported by a NHMRC Practitioner Fellowship (1 021 480). R.D. is supported by a NHMRC Practitioner Fellowship (1 059 157).

\section{DECLARATION OF INTEREST}

None.

\section{REFERENCES}

1. Britton PN, et al. Acute encephalitis in children: progress and priorities from an Australasian perspective. Journal of Paediatrics and Child Health 2015; 51: 147158.

2. Britton PN, et al. The causes and consequences of childhood encephalitis in Asia. Infectious Disorders Drug Targets 2014; 14: 78-88.

3. Stahl JP, Mailles A. What is new about epidemiology of acute infectious encephalitis? Current Opinion in Neurology 2014; 27: 337-341.

4. Coker RJ, et al. Emerging infectious diseases in southeast Asia: regional challenges to control. Lancet 2011; 377: 599-609.

5. Mackenzie JS. Emerging zoonotic encephalitis viruses: lessons from Southeast Asia and Oceania. Journal of Neurovirology 2005; 11: 434-440.

6. Wong KT. Emerging epidemic viral encephalitides with a special focus on henipaviruses. Acta Neuropathololgy 2010; 120: 317-325.

7. Zander A, et al. An outbreak of enterovirus 71 in metropolitan Sydney: enhanced surveillance and lessons learnt. Medical Journal of Australia 2014; 201: 663-666.

8. Solomon T, et al. Virology, epidemiology, pathogenesis, and control of enterovirus 71. Lancet Infectious Diseases 2010; 10: 778-790.

9. Morton NJ, et al. Severe hemorrhagic meningoencephalitis due to Angiostrongylus cantonensis among young 
children in Sydney, Australia. Clinical Infectious Diseases 2013; 57: 1158-1161.

10. Jones KE, et al. Global trends in emerging infectious diseases. Nature 2008; 451: 990-993.

11. Australian Bureau of Statistics. Canberra: Australian Bureau of Statistics, 2015. 3401.0-Overseas Arrivals and Departures, Australia, June 2015 (http://www.abs. gov.au/ausstats/abs@.nsf/products/961B6B53B87C130 ACA2574030010BD05). Accessed 2 January 2016.

12. Huppatz C, et al. Should there be a standardised approach to the diagnostic workup of suspected adult encephalitis? A case series from Australia. BMC Infectious Diseases 2010; 10: 353.

13. Huppatz C, et al. Etiology of encephalitis in Australia, 1990-2007. Emerging Infectious Diseases 2009; 15: 1359-1365.

14. Huppatz C, et al. Encephalitis in Australia, 1979-2006: trends and aetiologies. Communicable Disease Intelligence 2009; 33: 192-197.

15. Britton PN, et al. Encephalitis in Australian children: contemporary trends in hospitalisation. Archives of Disease in Childhood 2016; 101: 51-56.

16. Dalmau J, et al. Anti-NMDA-receptor encephalitis: case series and analysis of the effects of antibodies. Lancet Neurology 2008; 7: 1091-1098.

17. Irani SR, Vincent A. Autoimmune encephalitis-new awareness, challenging questions. Discovery Medicine 2011; 11: 449-458.

18. Tenembaum S, et al. Acute disseminated encephalomyelitis. Neurology 2007; 68 (16 Suppl. 2): S23-36.

19. Zurynski Y, et al. Paediatric active enhanced disease surveillance: a new surveillance system for Australia. Journal of Paediatrics and Child Health 2013; 49: 588-594.

20. Sejvar JJ, et al. Encephalitis, myelitis, and acute disseminated encephalomyelitis (ADEM): case definitions and guidelines for collection, analysis, and presentation of immunization safety data. Vaccine 2007; 25: 5771-5792.
21. Venkatesan A, et al. Case definitions, diagnostic algorithms, and priorities in encephalitis: Consensus Statement of the International Encephalitis Consortium. Clinical Infectious Diseases 2013; 57: 1114-1128.

22. Granerod J, et al. Causality in acute encephalitis: defining aetiologies. Epidemiology and Infection 2010; 138: 783-800.

23. Eastwood K, et al. Adult encephalitis surveillance: experiences from an Australian prospective sentinel site study. Epidemiology and Infection 2015; 143: 3300-3307.

24. Khatami A, et al. Sepsis-like disease in infants due to human parechovirus type 3 during an outbreak in Australia. Clinical Infectious Diseases 2015; 60: 228-236.

25. Cumming G, et al. Parechovirus genotype 3 outbreak among infants, New South Wales, Australia, 20132014. Emerging Infectious Diseases 2015; 21: 1144-1152.

26. Bernard S, et al. Epidemiology of infectious encephalitis, differences between a prospective study and hospital discharge data. Epidemiology and Infection 2013; 141: 2256-2268.

27. Pillai SC, et al. Infectious and autoantibody-associated encephalitis: clinical features and long-term outcome. Pediatrics 2015; 135: e974-984.

28. Granerod J, et al. Causes of encephalitis and differences in their clinical presentations in England: a multicentre, population-based prospective study. [Erratum appears in Lancet Infect. Dis. 2011; 11: 79.] Lancet Infectious Diseases 2010; 10: 835-844.

29. Britton PN, et al. Consensus guidelines for the investigation and management of encephalitis in adults and children in Australia and New Zealand. Internal Medicine Journal 2015; 45: 563-576.

30. Britton PN, et al. Consensus guidelines for the investigation and management of encephalitis. Medical Journal of Australia 2015; 202: 576-577.

31. Glaser CA, et al. Beyond viruses: clinical profiles and etiologies associated with encephalitis. Clinical Infectious Diseases 2006; 43: 1565-1577. 